\title{
Ein Gutachten als Werturteil des Sachverständigen
}

\author{
Landgericht Köln, Urteil vom 25. Februar 2013 - $240374 / 12$
}

Gutachten eines Sachverständigen können sowohl Tatsachenbehauptungen als auch Werturteile enthalten. Allerdings ist in der Regel der Schluss, den der Sachverständige in seinem Gutachten zieht, rechtlich in erster Linie ein Werturteil und nicht Behauptung einer Tatsache. (Leitsatz der Redaktion)

\section{Tatbestand}

- Die Parteien streiten um die Zulässigkeit einzelner Aussagen aus einem Gutachten des Beklagten zu 2) bzgl. der Echtheit dreier Bilder, welche von dem Maler Sigmar Polke stammen sollen.

Der Kläger bekam die Bilder von Herrn F aus Düsseldorf übergeben.

Der Kläger veräußerte die streitgegenständlichen Bilder an die Herren M, Kunstsammler aus Mönchengladbach, für einen Kaufpreis von 600.000 Euro.

Am 1. April 2011 begutachtete der Beklagte zu 2) die o.g. Bilder in Mönchengladbach. Am 15. April 2011 veröffentlichte er das Ergebnis seiner Untersuchung als Gutachten im Namen der Beklagten zu 1). Hinsichtlich der Einzelheiten des Gutachtens wird auf dieses Bezug genommen.

Der Vertrag wurde auf Wunsch der Käufer rückabgewickelt, nachdem diese das Gutachten des Beklagten zu 2) bzgl. der Bilder erhalten hatten.

Der Kunstsachverständige Prof. Dr. S begutachtete im Auftrag des Klägers die o.g. Bilder sowie das Gutachten des Beklagten zu 2) und fertigte am 17. Juni 2011 seinerseits ein Gutachten, wonach keine Zweifel an der Echtheit der Bilder bestünden.

Der Kläger ließ die o.g. Bilder bei Herrn Prof. Dr. B materialtechnisch überprüfen und drei Gutachten erstellen. Hinsichtlich des Inhalts der Gutachten wird auf diese Bezug genommen.

Mit Schreiben vom 7. Mai 2012, 10. Mai 2012 und 16. Mai 2012 forderte der Kläger die Beklagten auf, die Bezeichnung der o.g. Bilder als "nicht echt" zu unterlassen, solch getätigte Aussagen zu widerrufen und zu bestätigen, dass die Bilder anerkannt und in das zu erstellende Werksverzeichnis des Künstlers als echte Bilder aufgenommen würden.

Der Kläger begehrt mit seiner Klage die Unterlassung einzelner Teilaussagen aus dem Gutachten des Beklagten zu 2).

Der Kläger behauptet, er sei Eigentümer der o.g. Bilder. Er habe sie erworben vom Kunstspediteur F. Dieser habe Sigmar Polke gekannt und für diesen bis zu dessen Tod gearbeitet. Er habe über Jahrzehnte Bilder von Polke eingelagert und trans- portiert. In diesem Rahmen habe Polke dem Zeugen F drei Bilder versprochen und letzte auch übergeben. Die Bilder habe der Zeuge $\mathrm{F}$ gemeinsam mit dem Fahrer, dem Zeugen T abgeholt. Dieser habe dabei erklärt: „Das sind deine drei Bilder.“ Der Zeuge $A$, ein früherer Mitarbeiter Polkes, könne sich erinnern, dass zumindest eines der Bilder im Atelier Polkes vorhanden gewesen sei.

Der Zeuge F seinerseits habe die Bilder an den Kläger übereignet.

Der Geschäftsführer der L Kunstberatung $\mathrm{GmbH}$ habe die o.g. Bilder bei den Herren M gesehen und den Beklagten zu 2), welcher nicht für die Beklagte zu 1) aufgetreten sei, beauftragt, die Bilder zu begutachten. In der Folge sei versucht worden, die Herren $M$ für die gutachterliche Stellungnahme finanziell in Anspruch zu nehmen, was zurückgewiesen worden sei. In der Folge sei die L Kunstberatung $\mathrm{GmbH}$ in Anspruch genommen worden, was sich aus dem Schreiben des früheren Bevollmächtigten der Beklagten zu 1), Herr Rechtsanwalt Dr. C, ergebe.

Die Aussagen des Gutachtens des Beklagten zu 2) hinsichtlich der Maltechnik, der Holzrahmen, dem Alter der Bilder, der verwendeten Materialien und der Signaturen seien falsch, wie das Gutachten des Prof. Dr. S sowie die materialtechnischen Gutachten des Prof. Dr. B bewiesen. Die Summe der Falschfeststellungen des Beklagten zu 2) führe zu dem Ergebnis, dass auch die Gesamtbewertung des Beklagten zu 2) falsch sei.

Der Beklagte zu 2) sei kein Sachverständiger für Werke von Polke. Er habe Kenntnisse und Fähigkeiten nur vorgetäuscht und seine Untersuchung grob leichtfertig vorgenommen. Hierzu trägt der Kläger Näheres vor.

Das Gutachten vom 15. April 2011 erwecke den Eindruck einer vorgegebenen Auftragsarbeit.

Der Kläger beantragt,

1. die Beklagten zu verurteilen, es zu unterlassen, hinsichtlich dreier Bilder des Künstlers Sigmar Polke, deren Abbildungen dem Klageantrag als Abbildungen 1 bis 3 beigefügt sind, folgende Behauptungen aufzustellen:

a) Die insgesamt unqualifizierte Ausführung der Bildvorderseite entspricht in keinster Weise der künstlerischen Intention und der malerischen Qualität Sigmar Polkes. 
b) Das Nesselgewebe der Gemälde ist auf Keilrahmen des Künstlerbedarfs Typ Boesner, Typ Classic 45 mit einfachem Kreuz aufgespannt.

c) Es finden sich weder im Nachlass noch im bekannten Oeuvre vergleichbare Arbeiten.

d) Die Ausführung der drei Leinwände entspricht weitgehend dem Typus der in den vergangenen Monaten vor dem 1. April 2011 konfiszierten Falsifikate auf Papier und Gewebe, hier insbesondere zwei kleinerer Leinwände im Format $40 \times 50 \mathrm{~cm}$.

e) Zeichenkohle oder Schellack finden sich nicht in Sigmar Polkes Palette. Nachweisbar hat Polke nie Zeichenkohle bei seinen Leinwänden verwendet.

f) Die Signaturen stammen zweifelsfrei nicht von der Hand Sigmar Polkes.

g) Die drei Bilder sind quasi in einem Zug entstanden.

2. Für jeden Fall der Zuwiderhandlung haben die Beklagten eine Vertragsstrafe von jeweils 250.000 Euro zu zahlen.

3. Die Beklagten zu verurteilen, als Gesamtschuldner an den Kläger vorgerichtliche Kosten in Höhe von 7.868,28 Euro nebst Zinsen in Höhe von 5 Prozentpunkten über dem Basiszinssatz seit dem 22. Mai 2012 zu erstatten.

\section{Die Beklagten beantragen,}

die Klage abzuweisen.

Sie behaupten, der Beklagte zu 2) sei bei der Begutachtung ausschließlich im Namen der Beklagten zu 1) tätig geworden, nicht im eigenen Namen. Der Beklagte zu 2) sei daher nicht passivlegitimiert.

Das Gutachten des Beklagten zu 2) sei inhaltlich zutreffend. Die Beklagten verteidigen die angegriffenen Äußerungen im Einzelnen. Im Gegensatz dazu könne das Gutachten von Prof. Dr. S nicht überzeugen. Die materialtechnischen Gutachten stützten hinsichtlich der naturwissenschaftlichen Ergebnisse das Gutachten des Beklagten zu 2), in wesentlichen Punkten würden allerdings unzulässige und die Kompetenz des Gutachters Herrn Prof. Dr. B übersteigende Schlüsse gezogen.

Die Herren M hätten die L GmbH bzw. Herrn L eingeschaltet, um die Bilder einzuschätzen. Dieser habe die Bilder für falsch gehalten und zur Kontaktaufnahme mit dem Polke-Nachlass geraten.

Bei dem Gutachten handele es sich um eine Bewertung. Als solche sei sie keine von den Interessen der Beklagten gelenkte Auftragsarbeit, auch wenn sich die Beklagte zu 1) hinter die Bewertung gestellt habe.

Sie sind der Ansicht, es sei unzulässig, bei einer einheitlichen Gesamtbewertung der Bilder einzelne Unterlassungsanträge hinsichtlich einzelner Aussagen des Gutachtens zu stellen.
Eine Verpflichtung, Bilder als echt anzuerkennen, gebe es nicht, ebenso wenig eine Verpflichtung, die Bewertung der Bilder als falsch zurückzunehmen.

Ein Sachverständigengutachten enthalte in der Regel Werturteile und keine angreifbaren Tatsachen.

Selbst wenn die o.g. Bilder von Polke stammten, seien die Anträge zu 1) und 2) unzulässig.

Der Anspruch auf Zahlung einer Vertragsstrafe sei unbegründet.

Rechtsverfolgungskosten könne der Kläger auch deshalb nicht ersetzt bekommen, weil vorgerichtlich die Tätigkeit der Prozessbevollmächtigten des Klägers auf einen anderen Antrag gerichtet gewesen sei. Es sei um einen Anspruch auf Anerkennung der o.g. Bilder als echt und um die Aufnahme in das Werkverzeichnis von Polke gegangen sowie um die Unterlassung, die Bilder als unecht zu bezeichnen. Im hiesigen Verfahren solle aber die Kundgabe einzelner Aussagen des Gutachtens unterbunden werden.

Wegen der weiteren Einzelheiten des Sach- und Streitstandes wird auf die wechselseitigen Schriftsätze der Parteien nebst Anlagen Bezug genommen.

\section{Entscheidungsgründe}

Die Klage ist unbegründet.

Dem Kläger steht ein Unterlassungsanspruch gegen die Beklagten unter keinem rechtlichen Gesichtspunkt zu.

Dabei kann dahinstehen, ob der Beklagte zu 2) im eigenen Namen aufgetreten ist oder sein Gutachten nur als Stellungnahme der Beklagten zu 1) anzusehen ist. Soweit in dem vorliegenden Urteil von einem Gutachten des Beklagten zu 2) die Rede ist, soll damit nur zum Ausdruck gebracht werden, dass der Verfasser des Gutachtens der Beklagte zu 2) ist.

Ein Unterlassungsanspruch aus § 824 BGB scheitert am Fehlen von Tatsachenbehauptungen. Die angegriffenen Äußerungen stellen Werturteile, jedoch keine Tatsachenbehauptungen dar.

Gutachten eines Sachverständigen können sowohl Tatsachenbehauptungen als auch Werturteile enthalten. Allerdings ist in der Regel der Schluss, den der Sachverständige in seinem Gutachten zieht, rechtlich in erster Linie ein Werturteil und nicht Behauptung einer Tatsache. Es liegt im Wesen des Gutachtens, dass es auf der Grundlage bestimmter Verfahrensweisen zu einem Urteil kommt, das, selbst wenn es äußerlich als Tatsachenbehauptung formuliert worden ist, auf Wertungen beruht $(B G H$, Urteil vom 18. Oktober 1977 - VI ZR 171/76, zitiert nach [...], s. auch BGH, Urteil vom 9. Dezember 1975 - VI ZR 157/73, zitiert 
nach [...], sowie bereits RGZ 84, 294 ff.). Der Gutachter, der eine Untersuchung vorlegt und deren Ergebnisse darstellt, gibt nur seine subjektive Wahrnehmung und das daraus gewonnene Urteil wieder. Dem Wesen nach handelt es sich dann um die Kundgebung seiner subjektiven, gutachterlichen Überzeugung, die zwar angefochten und bestritten werden kann, auch unter dem Vorbehalt des Irrtums steht, aber immer ihrer Zielrichtung nach Wertung ist und von dem Empfänger auch so verstanden werden muss (BGH aaO, Rn. 37, zitiert nach [...]).

Bei Anwendung dieser Kriterien stellen die durch den Kläger angegriffenen Behauptungen des Beklagten zu 2) Werturteile und keine Tatsachenbehauptungen dar.

Die Äußerungen, die mit den Klageanträgen zu 1 a), c), d), f) und g) angegriffen werden, stellen bereits ihrem Wortlaut nach Bewertungen und keine Tatsachenbehauptungen dar.

Etwas anderes könnte allenfalls für die Äußerungen aus den Klageanträgen zu 1 b) und e) gelten. Es ist in der Rechtsprechung jedoch anerkannt, dass grundsätzlich auch Aussagen in Sachverständigengutachten, die wie Tatsachenbehauptungen klingen, als wissenschaftliche Stellungnahme und daher als Bewertung anzusehen sind.

Ausnahmsweise können sich Ausführungen in Gutachten allerdings dann als Tatsachenbehauptungen darstellen, wenn die der Schlussfolgerung vorausgehende methodische Untersuchung oder die zum Ergebnis führende Anwendung spezieller Kenntnisse und Fähigkeiten nur vorgetäuscht oder grob leichtfertig vorgenommen worden ist (BGH, Urteil vom 18. Oktober 1977 - VI ZR 171/76, Rn. 18, zitiert nach [...]).

Konkrete Anhaltspunkte hierfür bestehen allerdings nicht. Solche ergeben sich auch nicht aus der Tatsache, dass der Beklagte zu 2) das Gutachten im Namen der Beklagten zu 1) erstellt hat. Allein die Tatsache, dass es sich bei der Beklagten $z u$ 1) um die Erbengemeinschaft Polkes handelt, welche ihr legitimes Interesse an der autorisierten Zusammenstellung der Werke Sigmar Polkes vertreten, lässt nicht auf eine lediglich vorgetäuschte oder leichtfertig vorgenommene objektive methodische Untersuchung schließen. Anzeichen dafür, dass es sich um eine von den Interessen der Beklagten (fehl)gelenkte Auftragsarbeit handelt, bestehen nicht.

Ebenso führt das Anzweifeln der Sachkompetenz des Beklagten zu 2) nicht zu der o.g. Annahme. Zwar wird klägerseitig durchgehend die Sachkompetenz des Beklagten zu 2) angezweifelt und die Kompetenz des Gutachters Prof. Dr. S hervorgehoben, allerdings führt die Gegenüberstellung der beiden Gutachten nicht zu dem Schluss, dass die der Schlussfolgerung vorausgehende methodische Untersuchung oder die zum Ergebnis führende Anwendung spezieller Kenntnisse und Fähigkeiten nur vorgetäuscht oder grob leichtfertig vorgenommen worden ist. Der Gutachter des Klägers erklärt die streitgegenständlichen Bilder für "echt", während der Beklagte zu 2) sich an den Bildern „abarbeitet" und aufgrund seiner Feststellungen zumindest nachvollziehbare Schlüsse zieht. Prof. Dr. S, welchen der Kläger als Polke-Experten benennt, beurteilt das Gutachten des Beklagten zu 2) selbst und insbesondere dessen Herangehensweise nicht. Neben dem Ergebnis, die drei untersuchten Bilder seien echt, nimmt er keine Stellung zu dem ihm ebenfalls vorgelegten Gutachten des Beklagten zu 2). Aus den bloßen gegensätzlichen Ergebnissen der Begutachtungen lässt sich indes nicht der Schluss ziehen, der Beklagte zu 2) habe die der Schlussfolgerung vorausgehende methodische Untersuchung oder die zum Ergebnis führende Anwendung spezieller Kenntnisse und Fähigkeiten nur vorgetäuscht oder das Gutachten außerhalb jeder fachlichen Kompetenz erstellt. Es entspricht gerade dem Wesen der wissenschaftlichen Auseinandersetzung bei der Erstellung von Gutachten, dass diese oftmals zu unterschiedlichen Ergebnissen kommen, auch oder gerade aufgrund unterschiedlicher methodischer Ansätze. Wie der Kläger auf Seite 20 seiner Klageschrift selbst darlegt, sind „mit den Untersuchungen des Labors Prof. Dr. B und der Bewertung von Prof. S alle Erkenntnismittel zur Echtheit der Bilder erschöpft. Für die Beurteilung entscheidet letztlich wieder der Expertenblick." Aus diesen Gründen ist die Kammer nicht gehalten, ein Sachverständigengutachten einzuholen, welches die methodische Herangehensweise der von den Parteien vorgelegten Gutachten bewertet und einordnet.

Darüber hinaus haben die Beklagte zu 1) als Erbengemeinschaft nach dem Künstler Polke sowie der Beklagte zu 2) als künstlerischer Leiter der Beklagten zu 1) ein berechtigtes Interesse im Sinne des $\S 824$ Abs. 2 BGB an der wissenschaftlichen Auseinandersetzung mit dem künstlerischen Nachlass des Verstorbenen. Die Begutachtung einzelner Werke des Künstlers und die Erstellung schriftlicher Abhandlungen zu diesen Werken stellen sich als angemessene und legitime Mittel der Interessenwahrnehmung durch die Beklagte zu 1) dar. Das berechtigte Interesse des Beklagten zu 2) resultiert aus seiner Stellung als künstlerischer Leiter der Beklagten zu 1) und seinem wissenschaftlichen Interesse an der Auseinandersetzung mit dem Künstler Polke.

Ein Unterlassungsanspruch aus $\S \S 1004,823$ BGB analog scheidet ebenfalls aus.

Die angegriffenen Äußerungen stellen im Rahmen der Meinungsfreiheit des Art. 5 Abs. 1 GG zulässige Meinungsäußerungen dar. Da es sich bei den angegriffenen Äußerungen um Werturteile handelt (s.o.), ist der zulässige Raum für Meinungsäußerungen, welchen Art. 5 Abs.1 GG eröffnet, sehr weit. Es ließe sich mit dem Grundrecht auf Meinungsfreiheit nicht vereinbaren, den Verfasser eines solchen Gutachtens zur Unterlassung seiner subjektiven, auf seinen speziellen Kenntnissen, Erfahrungen und Untersuchungen beruhenden Überzeugung zu zwingen. Dies würde bedeuten, staatlicherseits den fachlichen Diskurs innerhalb der Kunstwissenschaft einzuschränken bzw. zu lenken. Dies kann und darf jedoch nicht staatliche Aufgabe sein, sondern muss durch gegenseitige Argumentation, Begut- 
achtung und Überzeugung geschehen. Es ist nicht Aufgabe dieses Gerichtes, Partei im wissenschaftlichen Meinungsstreit zu ergreifen. Es ist Aufgabe der interessierten Kreise, die Klärung der Urheberschaft der Werke von Sigmar Polke über Gutachten und Gegengutachten in einem freien Diskurs zu „erarbeiten."

Etwas anderes könnte sich nur ergeben, wenn die angegriffenen Äußerungen die Grenzen der Meinungsfreiheit überschritten. Dies wäre etwa dann der Fall, wenn sich die Äußerungen als "Schmähkritik" oder Formalbeleidigungen darstellten. Das Gutachten selbst ist jedoch sachlich gehalten und enthält keine persönlichen Vorwürfe, unzulässige Schmähkritik oder gar Formalbeleidigungen sind nicht zu erkennen. (Entscheidung von der Redaktion bearbeitet.) 